\title{
Transformación del Ejército Nacional, importancia del análisis de redes en su socialización
}

\section{Transformation of the National Army, importance of network analysis in its so- cialization}

\author{
Mikel I. Ibarra-Fernández \\ Universidad Externado de Colombia \\ ORCID iD: https://orcid.org/0000-0002-3984-8784 \\ Mikel.ibarra@uexternado.edu.co
}

\author{
Fecha de recepción: 13/02/2021 \\ Fecha de evaluación: 12/03/2021 \\ Fecha de aceptación: 10/06/2021 \\ Cómo citar: Ibarra-Fernández, M. (2021). Transfor-
mación del Ejército Nacional, importancia del análi-
sis de redes en su socialización. Revista Científica An-
fibios, $\quad 4(1) . \quad$ https://doi.org/10.37979/afb.2021v4n1.88
}

\section{Resumen}

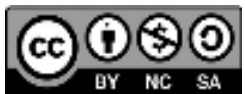

Atribución-NoComercial-CompartirIgual 4.0 Internacional

Transformar el principal pilar de la defensa nacional es uno de los objetivos que el Estado se traza y al cual dedica su mayor esfuerzo. El presente estudio tiene como principal objetivo indagar sobre el proceso de transformación de ejército nacional y analizar cómo se da la socialización del proceso de comunicación mediante redes. A metodología imprentada es de orden cualitativo, la técnica utilizada es análisis documental. La principal conclusión es que el análisis de redes puede aportar información relevante para el diseño de los planes de socialización de la transformación, tal como la identificación de los cargos con mayor centralidad e intermediación en la red que se configura con la estructura del Ejército.

\section{Palabras clave}

Institucionalismo; socialización; resistencia al cambio; transformación; análisis de redes

\begin{abstract}
Transforming the main pillar of national defense is one of the objectives that the State sets itself and to which it devotes its greatest effort. The main objective of this study is to investigate the transformation process of the national army and to analyze how the communication process is socialized through networks. The methodology used is qualitative, the technique used is documentary analysis. The main conclusion is that network analysis can provide relevant information for the design of transformation socialization plans, such as the identification of the positions with greater centrality and intermediation in the network that is configured with the structure of the Army.
\end{abstract}

Keywords

Institutionalism; socialization; resistance to change; transformation; network analysis 


\section{Introducción}

La transformación militar implica esfuerzos desde el punto de vista operacional y administrativo, pero "su implementación requiere cambios estructurales en la cultura, la filosofía y la ideología militar" (Ciro \& Correa, 2014, pág. 12); transcendiendo así a la perspectiva institucional.

El plan de transformación en el Ejército de Colombia requiere de un continuo proceso institucionalización-desinstitucionalización (Oliver, 1991) que permite cambiar prácticas y comportamientos arraigados en la institución; su efectividad depende en gran medida en el grado de aceptación y compresión que tienen sus miembros sobre él, facilitando así las modificaciones requeridas sobre los tres pilares de las instituciones definidos por Scott (1995). Desde la perspectiva de la justicia organizacional se afirma que, en el caso que las decisiones asociadas al cambio se consideren poco justas o adversas, la resistencia aumenta; y si en cambio, se consideran justas y promueven el bienestar son adoptadas más fácilmente (Hendrickson \& Gray, 2012); por lo tanto, la capacidad de socializar y difundir el plan transformación es determinante en su éxito.

El Ejército Nacional cuenta con cerca de 240.000 hombres distribuidos en la totalidad del territorio (Ejército Nacional, 2016), razón por la cual la capacidad de comunicar de forma eficiente el direccionamiento de la transformación, y clarificar su alcance y propósitos permite enfrentar la resistencia al cambio que se presenta en estos procesos. Lograr que el cambio no sea rechazado, y que se perciba como una oportunidad para que la institución prevalezca es un gran reto; vencer la resistencia al cambio es un factor crítico para la transformación ya que "menos de dos tercios de las iniciativas asociadas a este tema cumplen con las metas propuestas", Hendrickson \& Gray (2012, pág. 50) citando a Choi \& Ruona (2011). El análisis de redes puede brindar elementos determinantes para diseñar una estrategia integral de socialización en todos los niveles, permitiendo identificar aquellos cargos clave que deben emitir mensajes de forma permanente a todos los miembros de la institución sin importar su nivel jerárquico o área de operaciones. La carac- terización de la red podría mejorar el impacto de las estrategias de socialización y comunicación del direccionamiento de la transformación al interior de la fuerza mediante el estudio de distintos parámetros como las posiciones de sus nodos, la identificación de distancias, la centralidad, la intermediación, la cercanía y los clústeres presentes (Scott \& Davis, 2017).

En el año 2012 se dio inicio al plan de transformación del Ejército, y se han materializado importantes avances, los cuales se evidencian en hitos como la creación de la fuerza de tarea de armas combinadas, la actualización de la doctrina(damasco), la reorganización de estado mayor, el desarrollo de planes de formación y más de 3 procesos de socialización con cobertura nacional (Ejército Nacional, 2016); pese a esto, gran parte de los miembros de la institución desconocen el verdadero alcance de la transformación y en algunos casos, mal interpretan su propósito (Lagos, 2017). Para facilitar el proceso de difusión del Plan de Transformación es importante identificar cuáles son los cargos (en los niveles estratégico, operacional y táctico) que deben liderar las estrategias de comunicación del direccionamiento de la trasformación para impactar de forma eficiente a toda la red de dependencias y unidades que conforman al Ejército Nacional.

\section{Transformación del Ejército de Colombia desde la perspectiva institucional}

El Ejército de Colombia entiende la transformación militar como "una sucesión planeada de trabajos, esfuerzos e iniciativas tendientes a preparar la Fuerza para afrontar los retos venideros en los ambientes operacionales complejos" (Ciro \& Correa, 2014, pág. 21). La preparación del Ejército ante estas nuevas situaciones futuras requiere una permanente adaptación (Spencer, 2012), que afecta a los conceptos, capacidades, personas y organizaciones (Oana, Boscoinanu, Gabriela, Sumanaru, \& Anastasiei, 2011, pág. 1167).

La transformación institucional del Ejército se da como una respuesta a los cambios en el entorno, y conlleva un cambio organizacional que según Hendrickson \& Gray(2012, pág. 51) citando a Lines (2005) es "un cambio planeado en una estructura organizacional, sistemas 
o procesos que intenta lograr uno o más objetivos organizacionales"; para consolidar estos cambios se requiere según Alvarado Altamirano (2006, pág. 27) citando a Oliver (1992, pág. 564) que se generen procesos de desinstitucionalización "por los cuales la legitimidad de una práctica organizacional establecida o institucionalizada se erosiona o descontinúa, producto de la incapacidad demostrada por las prácticas vigentes para responder a nuevos desafíos determinados por presiones políticas, funcionales y sociales que se presentan tanto al exterior como al interior de la organización". De esta forma, según la visión institucionalista, la transformación sólo podrá consolidarse si es acompañada de procesos de desinstitucionalización de lo antiguo, que son seguidos de procesos de institucionalización de lo nuevo y requerido para hacer frente al futuro. Scott (1995) afirma que existen fuerzas que permiten trascender a lo institucional, las cuales se agrupan en tres pilares: regulativo que enmarca los aspectos asociados al monitoreo, control y sanción, normativo que rige el comportamiento social y finalmente, cultural-cognitivo que analiza la naturaleza de la realidad y los marcos que el ser humano utiliza para interpretarla y comprenderla; particularmente, la adecuada difusión de la transformación impacta el pilar cultural cognitivo, ya que les permite a los miembros de la fuerza entender las estrategias y nuevas directrices que se están implementando y que afectan sus actividades definidas en el pilar normativo.

Tabla 1 Pilares Institucionales y Portadores

\begin{tabular}{llll}
\hline Portador & Regulativo & Normativo & Cultural-Cognitivo \\
\hline Cultural & Reglas, Leyes & Valores, expectativas & Categorías, tipificaciones \\
\hline Estructuras sociales & $\begin{array}{l}\text { Sistemas de gobernanzas, } \\
\text { sistemas de poder }\end{array}$ & Sistemas de autoridad & Isomorfismo estructural, \\
& & & identidad
\end{tabular}

\begin{tabular}{llll}
\hline Rutinas & $\begin{array}{l}\text { Protocolos, procedimientos } \\
\text { estándar }\end{array}$ & $\begin{array}{l}\text { Conformidad, desem- } \\
\text { peño de las labores }\end{array}$ & $\begin{array}{l}\text { Programas para incentivar } \\
\text { el desempeño }\end{array}$
\end{tabular}

Fuente: Scott (1995, pág. 52)

La cultura organizacional es un concepto que según Frassa (2011, pág. 76) fue definido originalmente por Peters \& Waterman (1982) y Deal \& Kennedy (1982) mediante "una visión funcionalista de los aspectos simbólicos de la organización, concibiéndolos como objetos manipulables que pueden influir sobre la productividad y la competitividad de la empresa". Para cambiar o manipular deliberadamente la cultura organizacional para aportar a la transformación institucional del Ejército se requiere según Naranjo-Valencia \& Calderón-Hernández (2015, pág. 225) citando a Schein (1988) un conjunto de estrategias que permitan modificar el "modelo de presunciones básicas, inventadas, descubiertas o desarrolladas a medida que se fue aprendiendo a enfrentarse a los problemas de adaptación, y que han ejercido la suficiente influencia para ser consideradas válidas $\mathrm{y}$, en consecuencia, para ser enseñadas a los nuevos miembros como el modo correcto de percibir, pensar y sentir esos problemas". La modificación de la cultura facilita la aceptación del cambio disruptivo y permite desarrollar nuevas estrategias para enfrentar los desafíos del mañana (Prezelj, y otros, 2015).

El desarrollo y evolución del pilar cultural cognitivo se da como consecuencia de la presencia de formadores como "el contexto competitivo, el direccionamiento estratégico, la estructura, el liderazgo, los sistemas de medición y las prácticas de la gente" (Naranjo-Valencia \& Calderón-Hernández, 2015, pág. 226). 


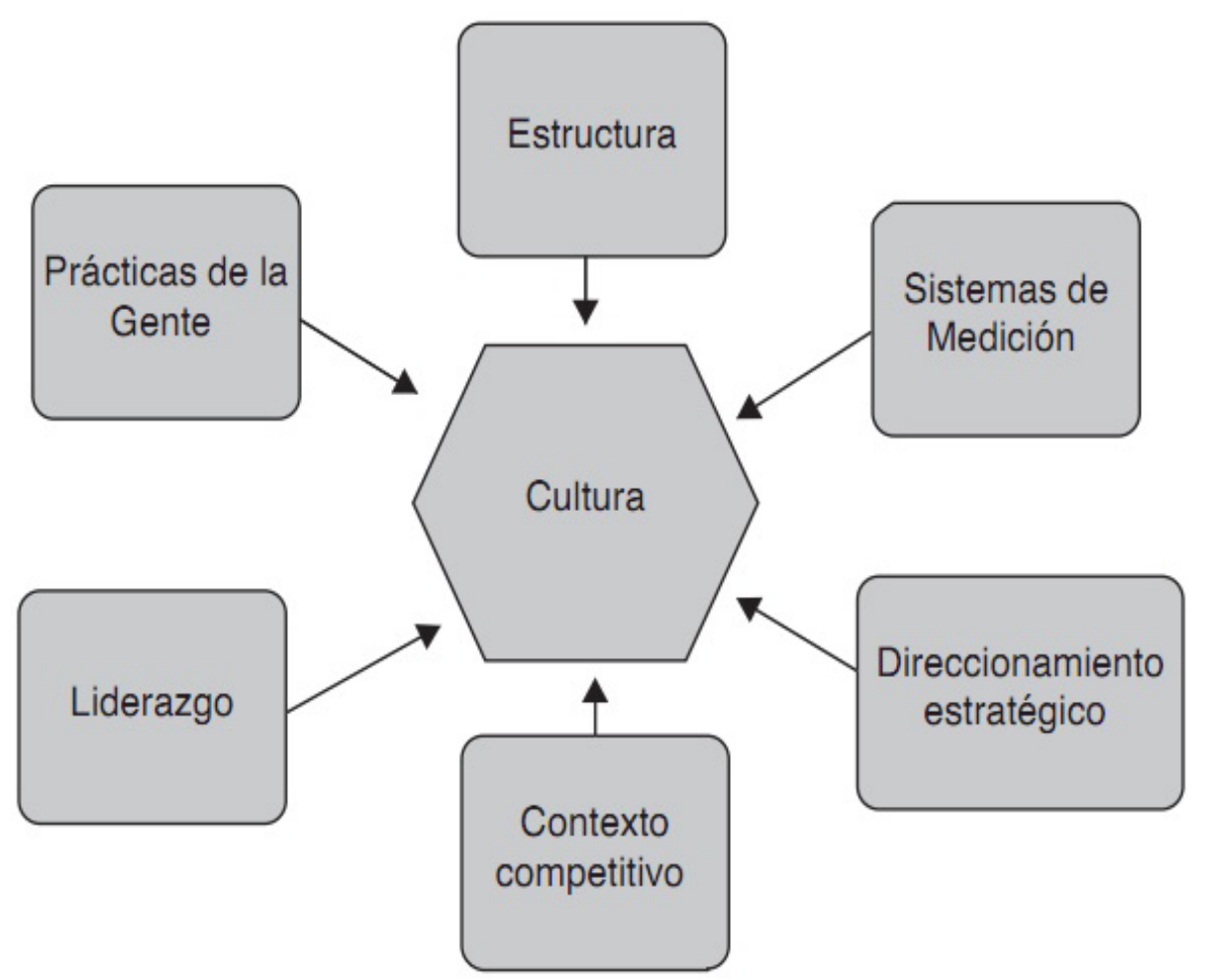

Ilustración 1 Formadores de Cultura

Fuente: (Naranjo-Valencia \& Calderón-Hernández, 2015, pág. 227)

De esta forma, la capacidad de socializar el direccionamiento estratégico, y particularmente el plan de transformación a toda la institución se convierte en un elemento clave para afectar el pilar cultural cognitivo, y en consecuencia potenciar los procesos de institucionalización y desinstitucionalización requeridos por la transformación. El General Gordon Sullivan reconocido transformador del Ejército de Estados Unidos de América afirmó que "la transformación del ejército se tenía que lograr mediante la acción positiva y agresiva, guiada por la visión y coherente con nuestros valores, acción que la gente pudiera entender" (Sullivan \& Harper, 1996, pág. 8)

\section{Posibles aportes del Análisis de redes en la socialización de la Transformación}

La estructura organizacional del Ejército puede ser analizada como una red, ya que sus tres niveles organizacionales (Estratégico: comando de fuerza, Operacional: dependencias que lideran los procesos, Táctico: unidades ejecutoras mayores (divisiones, brigadas) y menores batallones) interactúan de forma vertical y horizontal; dichas relaciones se encuentran de- finidas por el marco legal y la doctrina militar. La estructura organizacional actual es producto de una modificación del año 2016 (Ejército Nacional, 2016) y se encuentra dividida en cuatro grandes bloques:

Asesores de Comando y Segundo Comando: debe resaltarse particularmente el Comando de Transformación quien define el Plan de Transformación y por ende, las directrices estratégicas de mediano y largo plazo.

Jefatura de Planeación: despliega las directrices del Comando de Transformación, y está encargada de definir el direccionamiento de corto plazo en cada uno de los 11 procesos o subsistemas del Ejército mediante los departamentos Cede1 al Cede11 (estos subsistemas o procesos tienen cargos responsables en los niveles estratégico, operacional y táctico.). Este direccionamiento afecta a la Jefatura de Generador de Fuerza y a la Jefatura Operacional.

Jefatura de Generador de Fuerza y Jefatura Operacional: son las encargadas de operacionalizar las directrices de la Jefatura de Planeación, y lideran las divisiones y brigadas en todo el territorio nacional. 
Transformación del Ejército Nacional, importancia del análisis de redes en su socialización

\begin{tabular}{|c|c|}
\hline COEJEC & COMANDANTE DEL EJERCITO \\
\hline CEIGE & INSPECCION GENERAL COMANDO EJERCITO \\
\hline COTEF & COMANDO DE TRANSFORMACION DEL EJERCITO DEL FUTURO \\
\hline DICIE & DIRECCION DE CONTROL DE INVESTIGACIONES DEL EJERCITO \\
\hline OSMEJ & OFICINA SARGENTO MAYOR DE COMANDO EJERCITO \\
\hline SECEJ & SEGUNDO COMANDANTE DEL EJERCITO \\
\hline CEAYG & AYUDANTIA GENERAL COMANDO EJERCITO \\
\hline DIRIE & DIRECCION DE RELACIONES INTERNACIONALES DEL EJERCITO \\
\hline DANTE & DIRECCION APLICACIÓN DE NORMAS DE TRASNPARENCIA DEL EJERCITO \\
\hline COATE & COMANDO DE APOYO TECNOLOGICO DEL EJERCITO \\
\hline OADAS & OFICINA DE ASUNTOS DISCIPLINARIOS Y ADMINISTRATIVOS \\
\hline JEM PLANEACION Y POLITICAS & JEFE DE ESTADO MAYOR DE PLANEACION Y POLITICAS \\
\hline CEDE1 & COMANDO EJERCITO DEPARTAMENTO DE PERSONAL \\
\hline CEDE2 & COMANDO EJERCITO DEPARTAMENTO DE INTELIGENCIA Y CONTRAINTELIGENCIA \\
\hline CEDE3 & COMANDO EJERCITO DEPARTAMENTO DE OPERACIONES \\
\hline CEDE4 & COMANDO EJERCITO DEPARTAMENTO DE LOGISTICA \\
\hline CEDE5 & COMANDO EJERCITO DEPARTAMENTO DE PLANEACION \\
\hline CEDE6 & COMANDO EJERCITO DEPARTAMENTO DE COMUNICACIONES \\
\hline CEDE7 & COMANDO EJERCITO DEPARTAMENTO DE EDUCACION MILITAR \\
\hline CEDE8 & COMANDO EJERCITO DEPARTAMENTO FINANCIERO Y PRESUPUESTAL \\
\hline CEDE9 & COMANDO EJERCITO DEPARTAMENTO DE ACCION INTEGRAL Y DESARROLLO \\
\hline CEDE10 & COMANDO EJERCITO DEPARTAMENTO DE INGENIEROS \\
\hline CEDE11 & COMANDO EJERCITO DEPARTAMENTO DE JURIDICA INTEGRAL \\
\hline JEM GENERADOR DE FUERZA & JEFE ESTADO MAYOR DE APOYO DE FUERZA \\
\hline COPER & COMANDO DE PERSONAL \\
\hline COREC & COMANDO DE RECLUTAMIENTO \\
\hline CEDOC & COMANDO DE EDUCACION Y DOCTRINA \\
\hline COLOG & COMANDO DE LOGISTICA \\
\hline COING & COMANDO DE INGENIEROS \\
\hline JEM OPERACIONES & JEFE ESTADO MAYOR DE OPERACIONES \\
\hline CECAT & COMANDO ESPECIAL CONTRA AMENAZAS TRANSNACIONALES \\
\hline DIV & DIVISION (01-02-03-04-05-06-07-08) \\
\hline DAVAA & DIVISION DE AVIACION Y ASALTO AEREO \\
\hline CAIMI & COMANDO DE APOYO DE INTELIGENCIA MILITAR \\
\hline CACIM & COMANDO DE APOYO DE CONTRAINTELIGENCIA MILITAR \\
\hline CAOCC & COMANDO DE APOYO OPERACIONAL DE COMUNICACIONES Y CIBERDEFENSA \\
\hline CAAID & COMANDO DE APOYO DE ACCION INTEGRAL Y DESARROLLO \\
\hline CODES & COMANDO DE OPERACIONES DE DESPLIEGUE ESTRATEGICO \\
\hline FUDRA & FUERZA DE DESPLIEGUE RAPIDO (SEGREGADA FUERZA DE TAREA OMEGA) \\
\hline DIVFE & DIVISION FUERZAS ESPECIALES \\
\hline FUTAM & FUERZA DE TAREA DE ARMAS COMBINADAS \\
\hline FUDAI & FUERZA DE DESPLIEGUE DE APOYO INTERNACIONAL \\
\hline
\end{tabular}

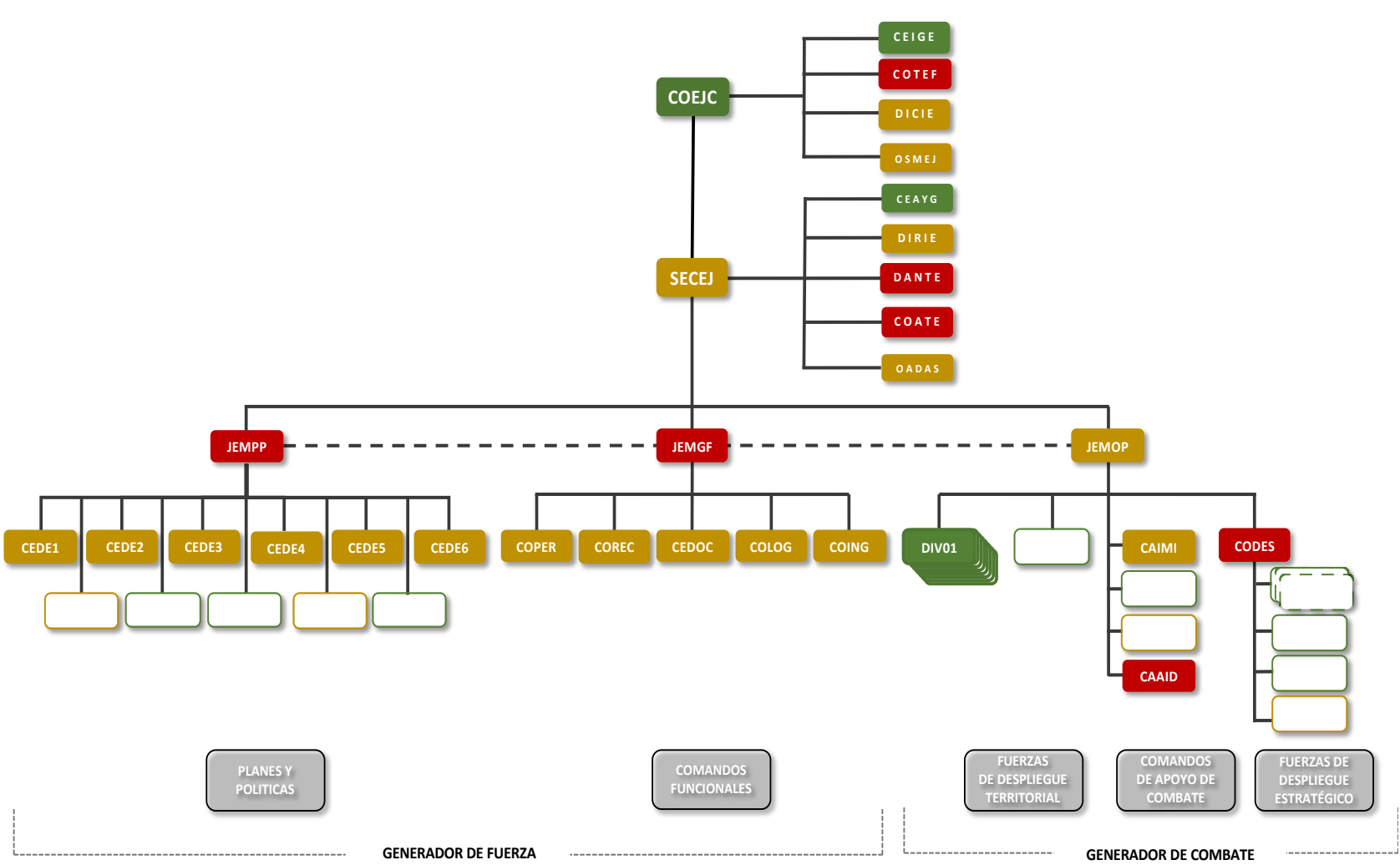

Ilustración 2 Estructura Organizacional Nivel Estratégico Fuente: (Ejército Nacional, 2016)

La estructura organizacional del nivel estra- de la especialización y características propias de tégico presentada se replica en los niveles opera- las unidades (Ejército Nacional, 2016). cional y táctico, con algunas diferencias producto 


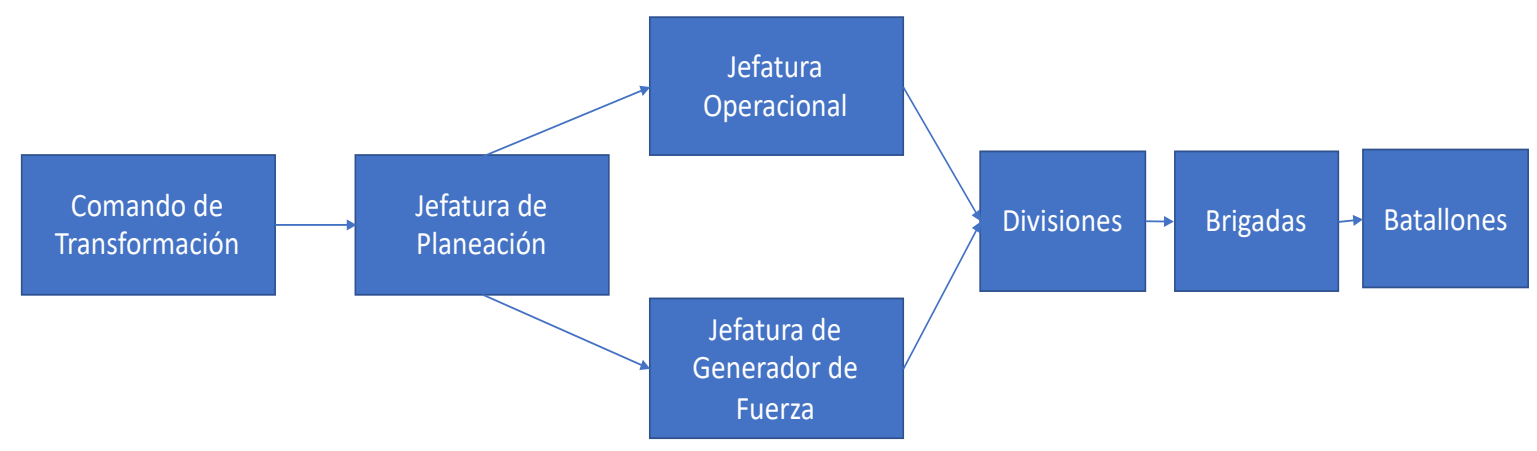

Ilustración 3 Niveles de Planeación y Transformación en la Estructura Organizacional Fuente: Elaboración Propia

Según Provan \& Kenis (2008), la gobernanza de redes implica "el uso de instituciones y estructuras de autoridad y colaboración para asignar recursos y para coordinar y controlar la acción conjunta en toda la red como un todo"; lo cual requiere una estrategia de comunicaciones que permita difundir eficientemente el direccionamiento de la institución. Debido a su complejidad, la estructura organizacional del Ejército podría ser analizada bajo la lógica de una red, y por lo tanto, el análisis de las interacciones entre los nodos se convertiría en un instrumento útil para identificar aquellos cargos que podrían influenciar eficientemente el comportamiento de los miembros de la institución.

Actualmente el Ejército Nacional cuenta con 8 divisiones territoriales, una división de aviación y una de fuerzas especiales; de allí se desprenden 40 brigadas, las cuales cuentan con una cifra de 6 a 8 batallones cada una (Ejército Nacional, 2016). Desde el año 2017 se encuentra en desarrollo un análisis para ajustar el número de batallones y rediseñar su estructura organizacional.

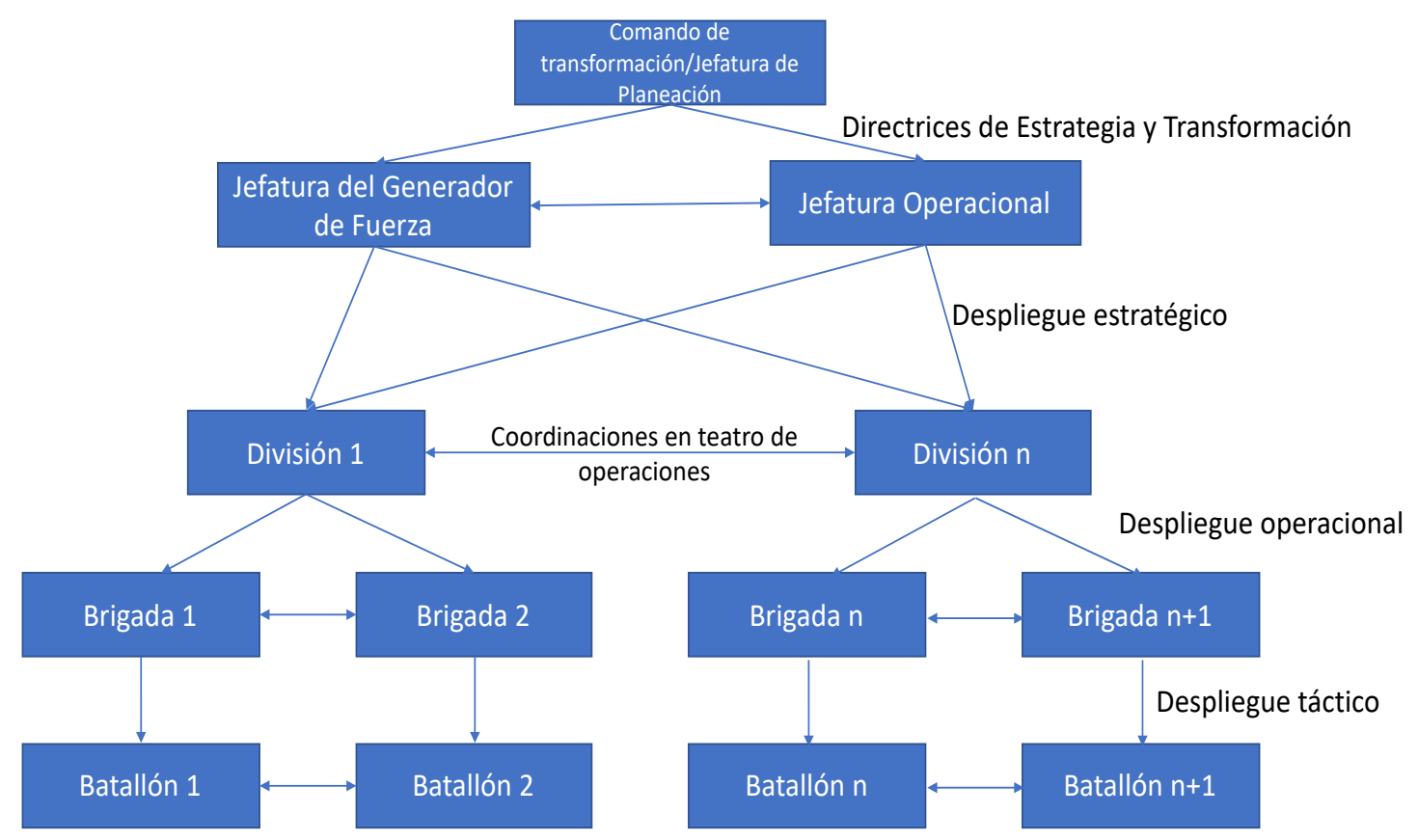

Ilustración 4 Estructura de la Red Ejército

Fuente: Elaboración Propia 
Según Scott \& Davis (2017) para caracterizar las redes, analizar sus nodos y sus relaciones se pueden usar algunos conceptos como:

las posiciones de sus nodos: identifica la forma en la que el actor o cargo se coordina con la topografía, permite aclarar si la posición del nodo es central o periférica.

la identificación de distancias: correspondiente al camino más corto que existe entre dos actores o nodos.

Intermediación: identificando que tan seguido un nodo se encuentra en el camino más corto entre otros dos nodos

la centralidad: identifica el nivel de importancia de un actor, usualmente se refiere a la cantidad de contactos directos que se tiene; de esta forma se puede identificar aquellos que más rápidamente pueden difundir mensajes, en este caso asociados a la transformación.

los clústeres presentes: identificando grupos de actores fuertemente conectados entre si, en forma de redes dentro de redes. De esta forma se pueden analizar si existen subgrupos que podrían presentar comportamientos diferenciales.

De esta forma el Ejército podrá identificar los cargos más relevantes para socializar el direccionamiento estratégico de la transformación a toda la institución, considerando factores como frecuencia de la comunicación y miembros que son impactados. El análisis de la comunicación en sistemas distribuidos y colaborativos permite el diseño de mejores mecanismos para favorecer el desempeño de las organizaciones $(\mathrm{Wu}$, Rosen, Panchal, \& Schaefer, 2015)processing, and sharing product design-related information. As engineering design processes are becoming increasingly distributed and collaborative, it is crucial to understand the communication and collaboration mechanism of engineers participating in such dispersed engineering processes. In particular, mapping initially disconnected design individuals and teams into an explicit social network is challenging. The objective of this paper is to propose a generic framework for investigating communication and collaboration mechanisms in social media-supported engineering design environments. Specifically, we propose an approach for measuring tie strengths in the con- text of distributed and collaborative design. We transform an implicit design network into an explicit and formal social network based on specific indices of tie strengths. We visualize the process of transforming customer needs to functional requirements, to design parameters, and to process variables using social network analysis (SNA.

El análisis de redes brinda un enfoque empírico para entender las comunicaciones interpersonales, permite movilizar a los individuos y favorecer la "adopción de las directrices" (Gainforth, Latimer-cheung, Athanasopoulos, Moore, \& Ginis, 2014, p. 2). Las posiciones de los actores en la red permiten crear corredores de comunicaciones que permiten optimizar la "comunicación entre personas desconectadas por especialización laboral" (Kawonga, Blaauw, \& Fonn, 2015, p. 7); de esta forma se facilita "la adopción de nuevas prácticas en las organizaciones mediante el intercambio de información a través de mecanismos interpersonales" (Gainforth et al., 2014, p. 5)

Particularmente, se podría mediante el análisis de redes verificar si los cargos de jefe de operaciones en las Brigadas son los nodos más relevantes para socializar el direccionamiento de la transformación; ya que su ubicación en la red como enlace entre las áreas de soporte (generador de fuerza) y las unidades operacionales los ubica en una posición central e impactan con su comunicación a la mayoría de miembros de la institución que se encuentran en el área de operaciones; además son los encargados de desplegar y monitorear los avances de los planes de corto, mediano y largo plazo emanados en el nivel estratégico.

\section{Conclusiones}

La transformación de las fuerzas militares se constituye en un esfuerzo institucional que usualmente parte de la necesidad de evolución debido a cambios en el entorno o ambiente operacional; pero el éxito en su desarrollo depende del nivel de entendimiento y compromiso que sus miembros tienen (Hansel \& Ruhnke, 2017, pág. 360); para lo cual es necesario garantizar que el direccionamiento provisto por la transformación sea desplegado de forma trasparente y con velocidad a todos los niveles de la organización (Ejército Nacional, 2012). 
El análisis de redes puede aportar información relevante para el diseño de los planes de socialización de la transformación, tal como la identificación de los cargos con mayor centralidad e intermediación en la red que se configura con la estructura del Ejército. De esta forma, se podrían diseñar mensajes y mecanismos adecuados para que la mayoría de la fuerza los conozca y comprenda rápidamente; también, la información de la red permitirá focalizar los cargos en donde se deben enviar personas con perfiles particulares para que cuenten con las competencias más ade- cuadas para socializar el plan de transformación mediante los mensajes y mecanismos diseñados para tal fin. Este documento es una invitación a la aplicación del análisis de redes a los planes de transformación militar, para focalizar esfuerzos y de esta forma vencer la resistencia al cambio e impactar efectivamente los pilares de las instituciones, acelerando los procesos de institucionalización y desinstitucionalización (Oliver, 1991), ejerciendo influencia sobre sus miembros a través de una visión de red.

\section{Referencias}

Alvarado Altamirano, S. (2006, agosto). Metamorfosis de la concepción del cambio organizacional en el nuevo institucionalismo. Contaduría y Administración (219), 11-40.

Ciro, A., \& Correa, M. (2014). Transformación estructural del Ejército colombiano. Construcción de escenarios futuros. General José María Córdova, 12(13), 19-88.

Collins, J., \& Futter, A. (2015). Reassessing the Revolution in Military Affairs: Transformation, Evolution and Lessons Learnt. Houndmills,: Palgrave Macmillan.

DiMaggio, P., \& Powell, W. (1983). The iron cage revisited: Institutional isomorphism and collective rationality in organizational Welds. American Sociological Review, 48: 147-60.

Ejército Nacional. (2012). Documento Estructural Comité Estratégico de Transformación e Innovación CETI. Bogotá: Ejército Nacional.

Ejército Nacional. (2014). Planeamiento por capacidades en el Ejército. Bogotá: Ejército.

Ejército Nacional. (2016). Documento Estructural Plan Estratégico de Transformación del Ejército del Futuro PETEF 1.0. Bogotá: Ejército.

Ejército Nacional. (2016). Informe Crei de Estructura. Bogotá: Ejército Nacional.

Everett, M., \& Borgatti, S. (2005). Extending Centrality. In P. Carrington, J. Scott, \& S. Wasserman, Models and methods In social networks analysis. Cambridge: Cambridge University.

Frassa, J. (2011, Julio). Cultura organizacional: conceptualizaciones y metodologías detrás de un concepto complejo. Dirección y Organización(44), 74-85.

Gainforth, H. L., Latimer-cheung, A. E., Athanasopoulos, P., Moore, S., \& Ginis, K. A. M. (2014). The role of interpersonal communication in the process of knowledge mobilization within a community-based organization: a network analysis, 1-8. https://doi.org/10.1186/1748-59089-59

Hair, J., Black, W., Babin, B., \& Anderson, R. (2009). Multivariate Data Analysis. New York: Prentice Hall.

Hendrickson, S., \& Gray, E. (2012, Marzo). Legitimizing Resistance to Organizational Change: A Social Work Social Justice Perspective. International Journal of Humanities and Social Science, 2(5), 50-59.

Ibarra, M. (2017). Fuerza multimisión: un efecto del plan de transformación del Ejército Nacional. Diálogo de las américas, 52-57. 
Kawonga, M., Blaauw, D., \& Fonn, S. (2015). Exploring the use of social network analysis to measure communication between disease programme and district managers atsub-national level in South Africa. Social Science and Medicine, 135, 1-14.https://doi.org/10.1016/j.socscimed.2015.04.024

Lagos, C. (2017, 12 04). Las dos orillas. Retrieved from Ruido de sables en el Ejército Nacional: https://www.las2orillas.co/ruido-de-sables-en-el-ejercito-nacional/

Mendeles, M. (2007). Military transformation past and present. London: Praeger.

Naranjo-Valencia, J., \& Calderón-Hernández, G. (2015). Construyendo una cultura de innovación. Una propuesta de transformación cultural. Estudios Gerenciales, 31, 223-236.

Norheim-Martinsen, P. (2016). New sources of military change - armed forces as normal organizations. Defence Studies, VOL. 16, NO. 3, 312-326.

Oana, P., Boscoinanu, M., Gabriela, P., Sumanaru, A., \& Anastasiei, T. (2011). MILITARY TRANSFORMATION IN THE CONTEXT OF GLOBALIZATION. Brasov: INTERNATIONAL CONFERENCE of SCIENTIFIC PAPER.

Ognyanova, K. (2016, 03 16). Kateto. Retrieved 09 2018, from Network Analysis and Visualization with R: http://www.kateto.net/wp-content/uploads/2016/01/NetSciX_2016_Workshop.pdf

Oliver, C. (1991). Strategic Responses to Institutional Processes. The Academy of Management Review, 16(1), 145-179.

Powell, W., \& DiMaggio, P. (1983). The Iron Cage Revisited: Institutional Isomorphism and Collective Rationality in Organizational Fields. American Sociological Review, 48:147-160.

Prezelj, I., Kopa, E., Vuga, J., Aleš , Ž., Kolak, A., \& Grizold, A. (2015). Military Transformation as Perceived by Experts. Journal of Slavic Military Studies, 28:23-47.

Provan, K., \& Kenis, P. (2008). Modes of Network Governance: Structure, Management, and Effectiveness. Journal of Public Administration Research \& Theory, 18(2), 229-252.

Roxborough, I. (2000). Organizational Innovation: lessons from military organizations. Sociological forum, 15, 367-372.

Sadegh Sharifirad, M., \& Ataei, V. (2010). Organizational culture and innovation culture: exploring the relationships between constructs. Leadership \& Organization Development Journal, 33(5), 494-517.

Scott, W. (1995). Institutions and Organizations. London: SAGE.

Scott, W. (2003). Organizations: Rational, Natural and Open Systems. Upper Saddle River, NJ: Prentice-Hall.

Scott, W. (2005). Institutional Theory. In K. SMITH, \& M. HITT, Greats minds in management (pp. 460-485). Oxford: Oxford University.

Scott, W., \& Davis, G. (2017). Organizations and organizing: rational, natural, and open system perspectives. Routledge.

Sloan, E. (2008). Military Transformation and Modern Warfare: A Reference Handbook. London: Praeger.

Spencer, D. (2012). Lessons from Colombia's Road to Recovery, 1982-2010. Strategic Issues in US/ Latin American Relations.

Sullivan, G., \& Harper, M. (1996). La esperanza no es un método. Bogotá: Norma. 
Szlechter, D. (2014). La cultura corporativa: una revisión crítica desde la Sociología del trabajo. Revista Venezolana de Gerencia, 19(65), 138 - 157.

Wasserman, S., \& Faust, K. (1994). Social Network Analysis: Methods and Applications. Methods and Applications. https://doi.org/10.1525/ae.1997.24.1.219

Wu, D., Rosen, D. W., Panchal, J. H., \& Schaefer, D. (2015). Understanding Communication and Collaboration in Social Product Development Through Social Network Analysis. Journal of Computing and Information Science in Engineering, 16(1), 011001. https://doi.org/10.1115/1.4031890 